\title{
Fast Fourier-Transform Second-Harmonic Generation (FFT-SHG) Provides a Solution for Measuring Nonlinear Effects on Fragile Structures
}

\author{
M. K. Vanbel ${ }^{\mathrm{a}}$, R. Paesen ${ }^{\mathrm{b}}$, W. Brullot ${ }^{\mathrm{a}}, \mathrm{S}$. Vandendriessche $^{\mathrm{a}}$, I. Asselberghs ${ }^{\mathrm{c}}$, K. Markey ${ }^{\mathrm{d}}$, P. Valvekens $^{\mathrm{d}}$, \\ M. A. van der Veen ${ }^{d}$, D. De Vos ${ }^{d}$, M. Ameloot ${ }^{b}$, V. K. Valev ${ }^{\text {e }}$ J.-P. Locquet ${ }^{f}$, T. Verbiest ${ }^{a}$ \\ ${ }^{a}$ Department of Chemistry, Celestijnenlaan 200D, B3001 Leuven, Belgium; ${ }^{b}$ BIOMED, University Hasselt and transnational \\ University Limburg, Agoralaan Building C, 3590 Diepenbeek, Belgium; ${ }^{c}$ Imec, Kapeldreef 75, 3001 Leuven, Belgium; ${ }^{d}$ Centre of \\ Surface Chemistry and Catalysis, Department of M2S, Kasteelpark Arenberg 23, B 3001 Leuven, Belgium; ${ }^{e}$ Cavendish Laboratory, \\ Department of Physics, University of Cambridge, J. J. Thomson Avenue, Cambridge CB3 OHE, United Kingdom; ${ }^{f}$ Department of \\ Physics and Astronomy, Celestijnenlaan 200D, B 3001 Leuven, Belgium \\ Authore-mail address: maarten.vanbel@chem.kuleuven.be
}

\begin{abstract}
The intense laser power required to measure nonlinear effects can damage the structures upon measuring. We demonstrate that fast Fourier-transform second-harmonic generation (FFT-SHG) technique provides an elegant solution to obtain polarization patterns of degrading structures.

OCIS code: (180.4315) Nonlinear microscopy; (190.4710) Optical nonlinearities in organic materials;
\end{abstract} (020.4180) Multiphoton processes

\section{Introduction}

Nonlinear optics is a broad research area with diverse research facets. Since the development of secondharmonic generation (SHG), which is a second-order nonlinear effect, the technique has been used to study structures in centrosymmetric materials by probing surface and interface specific properties such as adsorption of polymer films [1], chirality in polymer films [2], chirality in metamaterials [3], passivation of semiconductors [4], and diffusion of molecules into porous materials [5,6]. Besides the surface and interface properties, the crystal structure of a material can be obtained by analyzing the SHG polarization pattern which is typically measured by rotation of the polarization of the fundamental light and SHG detection through an analyzer [7]. One advantage of using SHG to probe the material crystal structure is that the SHG process itself does not require absorption of the photons to induce a real excited state [7]. The excited state is rather a virtual state where no energy transfer due to the incident photons occurs. Therefore, little damage is induced by the SHG experiment providing the possibility to probe fragile structures. However, when absorption at the fundamental frequency does occur, the SHG signal is enhanced due to resonance effects. A major drawback is that energy transfer is possible and heating of the structure is inevitable. This causes oxidation and breaking of bonds, resulting in structure degradation and ultimately leads to a drastic change in SHG response. Indeed, a full SHG characterization is usually done by recording the SH-intensity vs. input polarization for all SHG polarization components, leading to so called polarization curves. These polarization curves can be used to determine the symmetry of the sample. Since such measurements take a significant amount of time, degradation of the sample poses a serious problem. In analyzing the SHG polarization pattern, taking the damage induced intensity decay into account can be difficult since little is known on the degradation process. However, including the unknown decay component can be circumvented by performing a fast Fourier transform (FFT) to get information on only the different harmonic contributions, and by that the symmetry of a structure. An additional advantage of performing an FFT is that low signal to noise (SNR) data can still be used to obtain the required information. Therefore low SNR and fast measurements allow us to obtain sufficient data point before sample degradation becomes problematic. Finally, upon measuring the SHG response of structures by pointing a laser beam on the sample surface, a spatial average of the SHG response is detected. Any inhomogeneity in the sample cannot be probed separately and causes fluctuations in the SHG response when different areas of the same structure are probed. This problem can be overcome by combining the second-harmonic generation method with a microscope, in which only a limited area of the sample can contribute to the total signal. In this paper, we introduce microscope aided fast Fourier-transform Secondharmonic generation (FFT-SHG) analysis of chemical vapour deposited graphene, which has serious degradation issues, to obtain a polarization pattern of the structure. Moreover, the validity of the technique was confirmed with the metal-organic-framework (MOF) $\left[\mathrm{Ba}_{2} \mathrm{TMA}\left(\mathrm{NO}_{3}\right)(\mathrm{DMF})\right]$. By obtaining a polarization pattern by means of FFT-SHG, the issues with degradation of the graphene are resolved.

\section{Theory and experimental methods}

In general, the SHG polarization response can be written as 


$$
I(t)=\frac{a_{0}}{2}+a_{1} \sin \left(\omega_{0} t\right)+a_{2} \sin \left(2 \omega_{0} t\right)+a_{3} \sin \left(3 \omega_{0} t\right)+a_{4} \sin \left(4 \omega_{0} t\right)+\ldots
$$

where $a_{i}$ represents the amplitude of the $i^{\text {th }}$ harmonic in the FFT-SHG spectrum, and $\omega_{0}$ is the polarization modulation frequency set at $0.899 \mathrm{rad} / \mathrm{s}$. A possible phase shift is not taken into account since this does not contribute to the determination of the crystal structure. The FFT-SHG measurements were carried out on a commercial Zeiss multi-photon microscope consisting of a LSM510 mounted on an Axiovert 200M. The detection occurs in non-descanned (ND) mode using a photon counting detector (Hamamatsu H7422P-40). The photon counting detector is controlled using a Becker\&Hickl SPC830 FLIM system. The fundamental wavelength was set to $810 \mathrm{~nm}$ on the Mai Tai Deep See Laser (Spectra Physics) with a mean output power of $5 \mathrm{~mW}$. The polarization direction of the $810 \mathrm{~nm}$ fundamental beam is continuously rotated using an in house developed stepper motor controlled half wave plate which is contained in a slider and positioned directly under the microscope objective. The polarization modulation frequency is adjusted to the laser scanning speed to fulfil the Nyquist sampling theorem. The images are recorded with a 10x objective (NA of 0.3 ) and a pixel dwell time of $6.4 \mu \mathrm{s}$. A time series frame scanning is performed on a uniform region with a size of at least a few point spread functions (white rectangle Fig. 1a)), which in addition to the FFTSHG technique also slows sample deterioration. Every time frame is averaged into a single data point resulting in a time dependent SHG signal on which the FFT analysis is performed using Matlab's (The Mathworks) built in FFT function.

\section{Sample description}

A sheet of single layer CVD graphene is transferred from a $\mathrm{Cu}$ foil to a glass substrate by using classical transfer techniques $[8,9]$. Prior to graphene deposition, these glass slides are modified with location markers using standard photolithography. $\left[\mathrm{Ba}_{2} \mathrm{TMA}\left(\mathrm{NO}_{3}\right)(\mathrm{DMF})\right]$ (Ba MOF) was formed by combining $\mathrm{Ba}\left(\mathrm{NO}_{3}\right)_{2}, \mathrm{H}_{3}$ TMA and DMF at $423 \mathrm{~K}$. Details on the synthesis can be found elsewhere [10]. Foo et al. reported that the structure has a threefold rotation axis parallel to its $c$ axis and a twofold rotation axis parallel to its $b$ axis[10]. The crystals form colorless needles with their $b$ axis mostly parallel to the substrate surface.

\section{Discussion and results}

The graphene flake is located using SHG microscopy. After illumination, the structure exhibited a lower SHG response as compared to the first image revealing the problem of degradation of graphene samples. Therefore the proposed alternative FFT-SHG method is used to obtain a polarization pattern, and hence the symmetry of the sample. The area on which the FFT-SHG measurements were performed, are indicated with a white square (Fig. 1a). In the FFT-SHG spectrum of graphene (Fig. 1b), a large peak at $0 \mathrm{~Hz}$ can be observed and a small peak at $1 / 2 \omega_{0}$. According to Eq. 1, the first peak corresponds to the isotropic contribution of the SHG signal, which is frequency independent. The second peak occurs at the half of the polarization frequency, which is not expected to be present in the SHG polarization pattern. This peak is an artefact associated with the effective rotation speed of the half-wave plate. The polarization pattern can be calculated and is shown in Fig. 1c). For this, all frequencies except $1 / 2 \omega_{0}$ are taken into account. It can be seen that there are no features in the polarization pattern, which is expected for a hexagonal structure symmetry of the graphene sample [11].
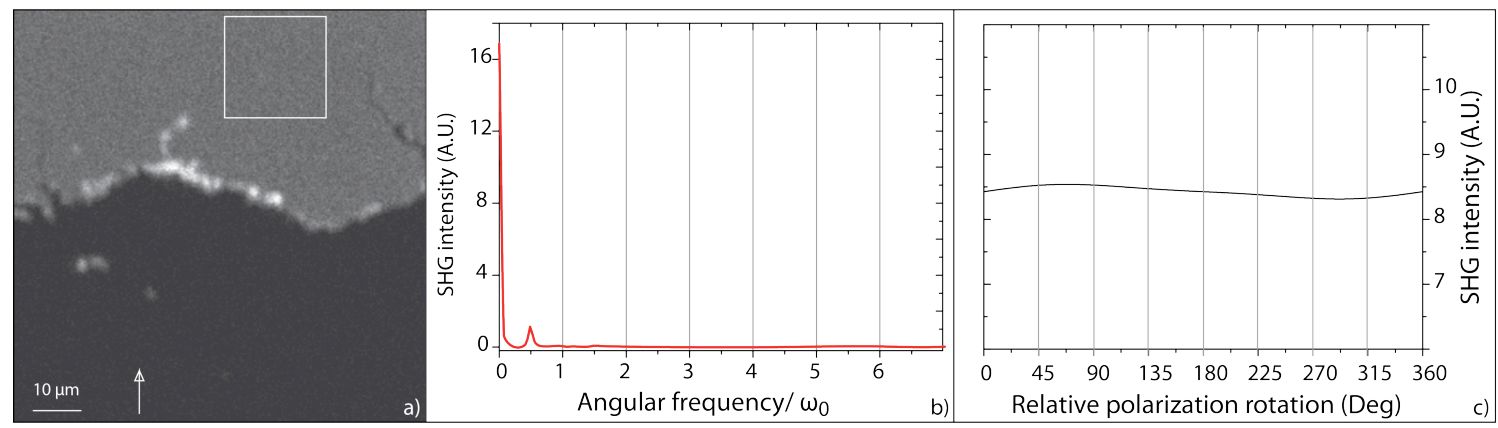

Figure 1: Polarization pattern determination of graphene. The location of the graphene layer was determined by secondharmonic generation microscopy (shown in a)). The arrow indicates the polarization direction of the fundamental beam before passing through the system for this figure. The highlighted white square is the region where the FFT-SHG measurements were performed. In b), the FFT-SHG spectrum of graphene is shown, displaying the possible harmonic contributions. From the FFT-SHG spectrum, the inverse polarization pattern with relative angle is calculated (shown in c)). All the parameters, but the frequency at $1 / 2 \omega_{0}$ were taken into account. This result is in agreement with the $\mathbf{C}_{\infty}$ symmetry of graphene. 
The Ba MOF needles were analysed in a similar manner as the graphene layer. This Ba MOF exhibits a constant SHG response under illumination. The structure is shown in Fig. 2a). A two fold symmetry is expected for this structure, since it has a two fold rotation axis and two symmetry planes in the structure. Two main peaks were observed in the FFT-SHG spectrum (Fig. 2b)), one at zero frequency and one at the double frequency. The extracted polarization pattern is shown in Fig. 2c). The obtained polarization pattern is compatible with the two-fold symmetry of the sample.
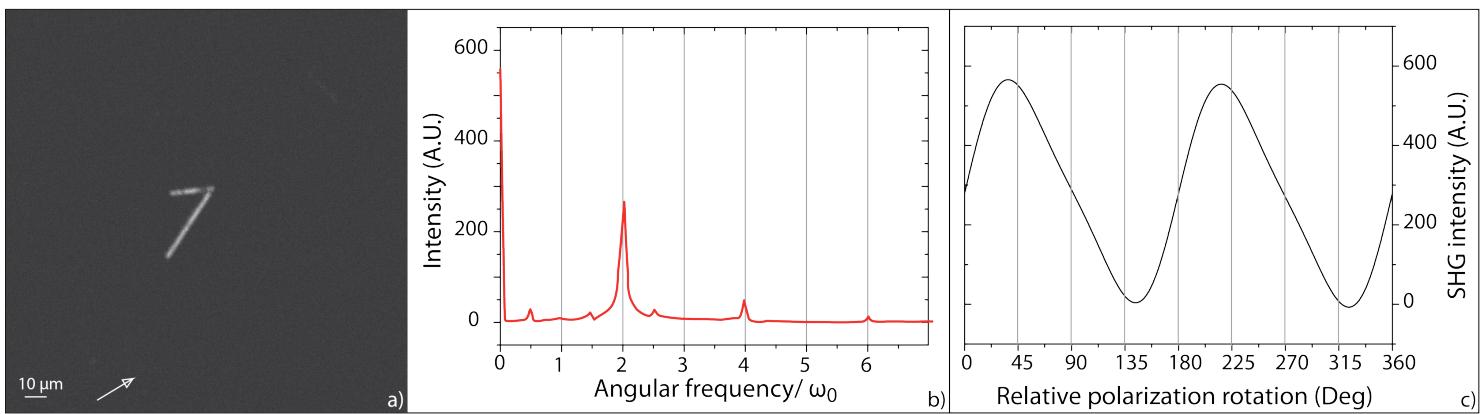

Figure 2: Polarization pattern determination of a Ba MOF needle. The location of the Ba MOF was determined by secondharmonic generation microscopy and two-photon fluorescence microscopy (shown in a)). The arrow indicates the polarization direction before passing through the system for this figure. In b), the FT-SHG spectrum is shown. The peaks in this spectrum determine the amplitude of the modulation at their respective frequencies. From this, the polarization pattern of the Ba MOF is reconstructed, which is shown in c). All the parameters, but the frequency at $1 / 2 \omega_{0}$ were taken into account.

\section{Conclusion}

We demonstrated that fast Fourier-transform second-harmonic generation (FFT-SHG) is an interesting technique for the study of fragile structures. SHG-polarization patterns can be obtained in a straightforward way, without problems with degradation of the sample.

\section{Acknowledgements}

This work was supported by the KU Leuven (GOA). We acknowledge the graphene sample preparation of Prof. B. J. Cho of KAIST Korea, Alexander Klekachev of imec and Frederik Drieskens. M.K.V. is grateful for the scientific discussions with M. Bloemen.

\section{References}

[1] C. Rotella, S. Napolitano, S. Vandendriessche, V. K. Valev, T. Verbiest, M. Larkowska, S. Kucharski and M. Wübbenhorst, "Adsorption Kinetics of Ultrathin Polymer Films in the Melt Probed by Dielectric Spectroscopy and Second-Harmonic Generation," LANGMUIR, 27, 13533-13538 (2011).

[2] T. Verbiest, S. Van Elshocht, A. Persoons, C. Nuckolls, K. E. Phillips and T. J. Katz, "Second-Order Nonlinear Optical Properties of Highly Symmetric Chiral Thin Films," LANGMUIR 17, 4685-4687 (2001).

[3] V. K. Valev, B. De Clerq, C. G. Biris, X. Zheng, S. Vandendriessche, M. Hojeij, D. Denkova, Y. Jeyaram, N. C. Panoiu, Y. Ekinci, A. V. Silhanek, V. Volskiy, G. A. E. Vandenbosch, M. Ameloot, V. V. Moshchalkov and T. Verbiest, "Distributing the Optical Near-Field for Efficient Field-Enhancements inNanostructures," Adv. Mater. 24, OP208-OP215 (2012).

[4] M. K. Vanbel, V. V. Afanas'ev, C. Adelmann, M. Caymax, V. K. Valev and T. Verbiest, "Tunneling of holes is observed by second-harmonic generation," Appl. Phys. Lett 102, 082104 (2013).

[5] M. A. van der Veen, V. K. Valev, T. Verbiest and D. E. De Vos, "In Situ Orientation-Sensitive Observation of Molecular Adsorption on a Liquid/Zeolite Interface by Second-Harmonic Generation,” LANGMUIR 25, 4256-4261 (2009).

[6] M. A. van der Veen, J. Van Noyen, B. F. Sels, P. A. Jacobs, T. Verbiest and D. E. De Vos, "Mappping of the organization of pnitroaniline in SAPO-5 by second-harmonic generation microscopy," Phys. Chem. Chem. Phys 12, 10688-10692 (2010).

[7] T. verbiest, K. Clays and V. Rodriguez, Second-order nonlinear optical characterization techniques (Taylor \& Francis Group, 2009), Chap. 2,3

[8] J. K. Park, S. M. Song, J. H. Mun and B.J. Cho, "Graphene Gate Electrode for MOS Structure-Based Electronic Devices" Nano Lett. 11, 5383-5386 (2011).

[9] T. Yoon, W. C. Shin, T. Y. Kim J.H. Mun, T.S. Kim and B.J. Cho, "Direct Measurement of Adhesion Energy of Monolayer Graphene As-Grown on Copper and its Application to Renewable Transfer," Nano Lett. 12, 1448-1452 (2012).

[10] M. L. Foo, S. Horike, Y. Inubushi and S. Kitagawa, "Alkaline Earth I3O0 Porous Coordination Polymer:

[Ba2TMA(NO3)(DMF)]**," Angew. Chem. Int. Ed. 51, 6107-6111 (2012).

[11] M. A. van der Veen, F. Vermoortele, D. E. De Vos and T. Verbiest, "Point Group Symmetry Determination via Observables Revealed by Polarized Second-Harmonic Generation Microscopy: (1) Theory,” Anal. Chem. 84, 6378-6385 (2012). 